\title{
Defects at nitrogen site in electron-irradiated AIN
}

Nguyen Son Tien, A. Gali, A. Szabo, M. Bickermann, T. Ohshima, J. Isoya and Erik Janzén

\section{Linköping University Post Print}

N.B.: When citing this work, cite the original article.

Original Publication:

Nguyen Son Tien, A. Gali, A. Szabo, M. Bickermann, T. Ohshima, J. Isoya and Erik Janzén, Defects at nitrogen site in electron-irradiated AlN, 2011, Applied Physics Letters, (98), 24, 242116.

http://dx.doi.org/10.1063/1.3600638

Copyright: American Institute of Physics

http://www.aip.org/

Postprint available at: Linköping University Electronic Press

http://urn.kb.se/resolve?urn=urn:nbn:se:liu:diva-69860 


\title{
Defects at nitrogen site in electron-irradiated AIN
}

\author{
N. T. Son, ${ }^{1, a)}$ A. Gali, ${ }^{2,3}$ Á. Szabó, ${ }^{3}$ M. Bickermann, ${ }^{4}$ T. Ohshima, ${ }^{5}$ J. Isoya, ${ }^{6}$ and \\ E. Janzén ${ }^{1}$ \\ ${ }^{1}$ Department of Physics, Chemistry and Biology, Linköping University, SE-581 83 Linköping, Sweden \\ ${ }^{2}$ Research Institute for Solid State Physics and Optics, Hungarian Academy of Sciences, P.O. Box 49, \\ H-1525 Budapest, Hungary \\ ${ }^{3}$ Department of Atomic Physics, Budapest University of Technology and Economics, Budafoki út 8, \\ H-1111 Budapest, Hungary \\ ${ }^{4}$ Department of Materials Science 6, University of Erlangen-Nürnberg, Martensstrasse 7, \\ D-91058 Erlangen, Germany \\ ${ }^{5}$ Japan Atomic Energy Agency, 1233 Watanuki, Takasaki, Gunma 370-1292, Japan \\ ${ }^{6}$ Graduate School of Library, Information and Media Studies, University of Tsukuba, Tsukuba, \\ Ibaraki 305-8550, Japan
}

(Received 14 April 2011; accepted 25 May 2011; published online 17 June 2011)

\begin{abstract}
In high resistance AlN irradiated with $2 \mathrm{MeV}$ electrons, an electron paramagnetic resonance (EPR) spectrum, labeled EI-1, with an electron spin $S=1 / 2$ and a clear hyperfine (hf) structure was observed. The hf structure was shown to be due the interaction between the electron spin and the nuclear spins of four ${ }^{27} \mathrm{~A}$ nuclei with the hf splitting varying between $\sim 6.0$ and $\sim 7.2 \mathrm{mT}$. Comparing the hf data obtained from EPR and ab initio supercell calculations we suggest the EI-1 defect to be the best candidate for the neutral nitrogen vacancy in AlN. (C) 2011 American Institute of Physics. [doi:10.1063/1.3600638]
\end{abstract}

In recent years, considerable progress in crystal growth and in $n$-type and $p$-type doping of aluminum nitride (AIN) has led to the fabrication of light emitting diodes in deep ultraviolet (UV) spectral region ${ }^{1}$ and made the material more promising for deep-UV laser applications. However, doping is still a serious problem for AlN and its alloy AlGaN with high $\mathrm{Al}$ content. Nominal undoped AlN grown by either metalorganic chemical vapor deposition or physical vapor transport (PVT) is semi-insulating. This is generally believed to be due to carrier compensation by deep level defects such as residual oxygen at $\mathrm{N}$ site $\left(\mathrm{O}_{\mathrm{N}}\right)$ and/or the $\mathrm{N}$ vacancy $\left(\mathrm{V}_{\mathrm{N}}\right)$ donor centers in the case of $p$-type doping or the $\mathrm{Al}$ vacancy $\left(\mathrm{V}_{\mathrm{Al}}\right)$ acceptor center in the case of $n$-type doping. The $\mathrm{V}_{\mathrm{N}}$ (or $\mathrm{V}_{\mathrm{Al}}$ ) has been predicted by theory to have low formation energies in $p$-type (or $n$-type) AlN and is expected to be abundant in as-grown materials. ${ }^{2-4}$ The calculations also suggested that $\mathrm{V}_{\mathrm{N}}$ transforms from a shallow donor in $\mathrm{GaN}$ to a deep donor in AlN and compensates acceptors, making $p$-type doping of AlN difficult. So far, no conclusive experimental identification of vacancies in AlN has been reported. In neutron-irradiated polycrystalline $\mathrm{AlN}$, a broad electron paramagnetic resonance (EPR) signal with $g=2.007$ was assigned to $\mathrm{V}_{\mathrm{N}}{ }^{5,6}$ In as-grown AlN, a number of optical detection of EPR spectra were observed but non of them showed a resolved hyperfine (hf) structure and hence could not be identified. ${ }^{7}$ In a more recent study of as-grown AlN, an EPR spectrum with an unresolved hf structure due to the interaction with ${ }^{27} \mathrm{Al}$ nuclei (nuclear spin $\mathrm{I}=5 / 2$ and $100 \%$ natural abundance) was observed and assigned to either the neutral $\mathrm{N}$ vacancy $\left(\mathrm{V}_{\mathrm{N}}^{0}\right)$ or the shallow $\mathrm{O}_{\mathrm{N}}$ donor. ${ }^{8}$

In this letter, we report our observation of an EPR spectrum with an electron spin $S=1 / 2$ and a clear hf structure in AlN after electron irradiation. The structure is shown to be due to the hf interaction between the electron spin and the

${ }^{\text {a)} E l e c t r o n i c ~ m a i l: ~ s o n @ i f m . l i u . s e . ~}$ nuclear spins of four ${ }^{27} \mathrm{Al}$ nuclei. Comparing the ${ }^{27} \mathrm{Al}$ hf data obtained from EPR and ab initio calculations we suggest the defect to be the best candidate for $\mathrm{V}_{\mathrm{N}}^{0}$ in AlN.

The samples used in our study are bulk AlN grown by PVT. The irradiation with $2 \mathrm{MeV}$ electrons was performed at $\sim 300 \mathrm{~K}$ with doses $\sim 2-10 \times 10^{18} \mathrm{~cm}^{-3}$. EPR measurements were formed on X-band $(\sim 9.5 \mathrm{GHz})$ Bruker E500 and E580 spectrometers using a continuous flow cryostat, allowing sample temperature regulation in the range 4$295 \mathrm{~K}$.

In as-grown $\mathrm{AlN}$, an isotropic EPR signal at $\sim 338 \mathrm{mT}$ corresponding to a g-value of $\sim 2.009$ was observed in darkness in a wide range of temperature (4-295 K). Figure 1(a) show this spectrum measured at $20 \mathrm{~K}$ for the magnetic field along the c-axis $(\mathbf{B} \| \mathbf{c})$. After electron irradiation, this line

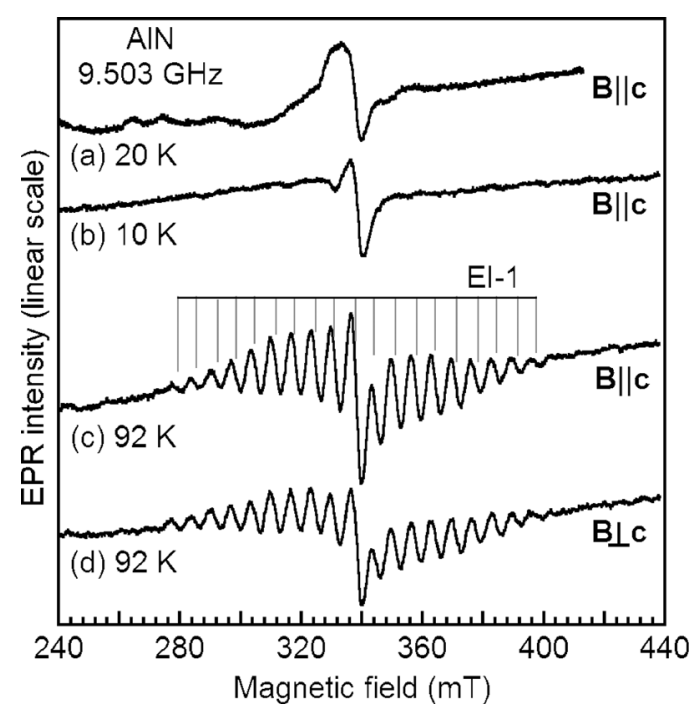

FIG. 1. EPR spectra observed in darkness (a) in as-grown AlN and (b-d) in electron-irradiated AlN at different temperatures. The isotropic signal at $\sim 338 \mathrm{mT}$ in (a) and (b) is from an unidentified defect. 


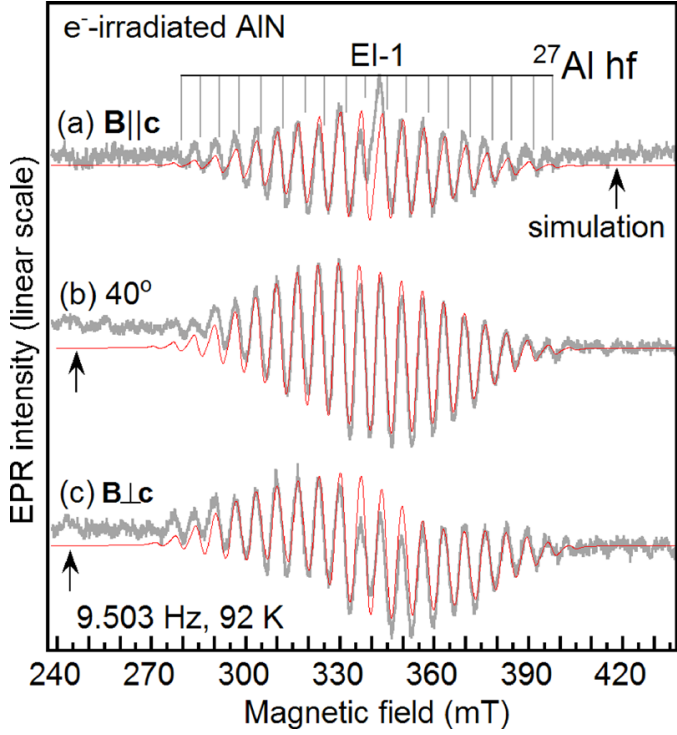

FIG. 2. (Color online) EPR spectra (in gray) measured in irradiated AlN in darkness at $92 \mathrm{~K}$ for (a) $\mathbf{B} \| \mathbf{c}$, (b) $\mathbf{B}$ is $40^{\circ}$ off the c-axis, and (c) $\mathbf{B} \perp \mathbf{c}$ after subtracting the isotropic signal in Fig. 1(b). The simulated spectra (indicated by arrows) assuming the hf interaction with two pairs of equivalent ${ }^{27} \mathrm{Al}$ neighbors having the corresponding hf splittings of: (a) $6.5 \mathrm{mT}$ and $6.7 \mathrm{mT}$, (b) $6.5 \mathrm{mT}$ for one pair of ${ }^{27} \mathrm{Al}$ neighbors and $6.0 \mathrm{mT}$ and $7.2 \mathrm{mT}$ for each of other two ${ }^{27} \mathrm{Al}$ atoms, and (c) $6.0 \mathrm{mT}$ and $7.05 \mathrm{mT}$ for each pairs. The spectra were simulated with $\mathrm{g}=2.012$ and the Gaussian line shape with a line width of $2.4 \mathrm{mT}$

does not seem to be affected but the broad signal overlapping with this line at the low-field side is reduced [Fig. 1(b)]. At temperatures above $80 \mathrm{~K}$, a new spectrum, labeled EI-1 (EI: electron irradiation), with a hf structure consisting of $19 \mathrm{hf}$ lines of nearly equal splitting $(\sim 6.6 \mathrm{mT})$ was observed. Figures 1(c) and 1(d) show this spectrum measured in darkness at $92 \mathrm{~K}$ for $\mathbf{B} \| \mathbf{c}$ and $\mathbf{B} \perp \mathbf{c}$, respectively.

Figure 2 shows the measured spectra (in gray) for different directions of the magnetic field as follows: (a) $\mathbf{B} \| \mathbf{c}$ (denoted as $0^{\circ}$ ), (b) $\mathbf{B}$ is $40^{\circ}$ off the c-axis, and (c) $\mathbf{B} \perp \mathbf{c}\left(90^{\circ}\right)$. In these spectra, the isotropic signal at $\sim 338 \mathrm{mT}$ shown in Fig. 1(b) was subtracted. The angular dependence study with B rotating in the $(11 \overline{2} 0)$ plane shows that the hf lines are nearly isotropic but their relative peak intensity changes with the angle of the magnetic field as can be seen in Figs. 2(a)-2(c). The spectrum in Fig. 2(a) shows a rhombic shaped envelope typical for a $\mathrm{hf}$ interaction with a number of equivalent nuclei. In AlN, both ${ }^{14} \mathrm{~N}(\mathrm{I}=1,100 \%$ natural abundance) and ${ }^{27} \mathrm{Al}$ have nonzero nuclear spins. Our simulation shows that the hf interaction with four equivalent ${ }^{27} \mathrm{Al}$ nuclei with $\mathrm{I}=5 / 2$ gives rise to $21 \mathrm{hf}$ lines with the intensity ratio: $1: 4: 10: 20: 35: 45: 79: 103: 124: 140: 144: 140: 124: 103: 79$ : $45: 35: 20: 10: 4: 1$. This gives rise to a hf structure with the rhombic shaped envelope. The largest splitting lines with the intensity ratio of 1 are too weak to be distinguished from the noise level and therefore the observed spectra show $19 \mathrm{hf}$ lines. The hf interaction with four equivalent ${ }^{14} \mathrm{~N}$ nuclei (I $=1$ ) would result in only $9 \mathrm{hf}$ lines. We therefore believe that the EI-1 spectrum is related to a defect at $\mathrm{N}$ site and has the $\mathrm{hf}$ interaction with four nearest ${ }^{27} \mathrm{Al}$ neighbors. Since only the peak intensity of the $\mathrm{hf}$ lines and the envelope shape of the hf structure are markedly changed with the magnetic field direction while the angular variation in the line position is small (see Fig. 2), we can conclude that the anisotropy of the hf interaction should be smaller than the line width $(\sim 2.6 \mathrm{mT})$. In this case, the anisotropy of the $\mathrm{hf}$ interaction could not be studied from the angular dependence of the line position but can still be estimated from fitting of the hf structures at different crystal directions. The angular variation in EPR lines caused by the anisotropy of the g-value is within the line width and cannot be determined. Since the anisotropy of the g-value of intrinsic defects in semiconductors is usually small ( $\sim 0.1 \%$ or less) we neglected the anisotropy of the g-value and used the value corresponding to the center of the hf structure $(\mathrm{g}=2.012)$ in the hf fitting.

In the case of a $C_{3 v}$ center, the $\mathrm{Al}$ atom along the c-axis moves away from the vacancy site and the spin density located in the dangling bond of this atom is often larger than that at one of the other three equivalent atoms in the basal plane. For $\mathbf{B} \| \mathbf{c}$, it is expected to observe two sets of hf lines corresponding to the interaction (i) with one $\mathrm{Al}$ atom along the c-axis having $C_{3 v}$ symmetry with a larger hf splitting and (ii) with three equivalent $\mathrm{Al}$ atoms in the basal plane having $C_{1 h}$ symmetry with smaller hf splittings. In the case of a $C_{1 h}$ center, reconstructed bonds are formed and the hf interactions with the pairs of atoms in the basal or vertical planes also give rise to two sets of hf lines both having $C_{1 h}$ symmetry. However, without dangling bonds, there is no atom with preferential spin localization and the hf splittings are expected to be less anisotropic. We found that the hf structure at $\mathbf{B} \| \mathbf{c}$ can be fitted if hf splittings are in the range 6.5-6.7 mT for $C_{1 h}$ symmetry or $6.5-6.9 \mathrm{mT}$ for $C_{3 v}$ symmetry. Indeed, the hf structure in Fig. 2(a) has an envelope close to a rhombic shape. The simulated spectrum for $C_{1 h}$ symmetry with splitting of 6.5 and $6.7 \mathrm{mT}$ for two pairs of equivalent $\mathrm{Al}$ atoms is shown in Fig. 2(a). With increasing the hf splitting of one $\mathrm{Al}$ atom from 6.9 to $7.2 \mathrm{mT}$ (for $C_{3 v}$ symmetry), the deviation in line position of outer hf lines increases to $0.5-0.7 \mathrm{mT}$. This also causes a deviation in the intensity ratio of hf lines from the case of the interaction with four equivalent $\mathrm{Al}$ neighbors and the simulated spectrum shows a clear parallelogram-shaped envelope similar to the spectrum in Fig. 2(b). For $\mathbf{B} \perp \mathbf{c}$, the $C_{1 h}$ symmetry should give rise to two sets of hf lines for each pair and in total four sets of hf lines are expected. However, we found that the spectra can be very well fitted with two sets of hf lines with the splitting of 6.0 and $7.05 \mathrm{mT}$ [Fig. 2(c)]. Using four sets of hf lines with $0.1-0.2 \mathrm{mT}$ different in the hf splitting, i.e., $6.0 \pm(0.1-0.2) \mathrm{mT}$ and $7.05 \pm(0.1-0.2) \mathrm{mT}$, does not improve the fit. The fit gets worse when changing the splitting of the two sets by more than $0.2 \mathrm{mT}$. This indicates that the difference in hf splitting of two pairs of $\mathrm{Al}$ atoms is within $\sim 0.2 \mathrm{mT}$. In the intermediate angles between $0^{\circ}$ and $90^{\circ}$ in the $(11 \overline{2} 0)$ plane, each $\mathrm{hf}$ line will split into four lines due to $C_{1 h}$ symmetry. Assuming that the two sets of the hf lines corresponding to two pairs of $\mathrm{Al}$ atoms are also similar (within $0.2 \mathrm{mT}$ ), we could fit all the observed spectra. Figure 2(b) shows the simulated spectrum for the angle of $40^{\circ}$ off the c-axis using the hf splitting of: $6.5 \mathrm{mT}$ for double sites (corresponding to the interaction with two $\mathrm{Al}$ atoms) and $6.0 \mathrm{mT}$ and $7.2 \mathrm{mT}$ for each single site (corresponding to the interaction with one $\mathrm{Al}$ atom). These ${ }^{27} \mathrm{Al}$ hf splittings, 6.0 and $7.2 \mathrm{mT}$, were found to be the smallest and largest, respectively, among the observed values. We can therefore estimate the principal values of the hf tensors of the two pairs of $\mathrm{Al}$ atoms to be about: $\mathrm{A}_{\mathrm{xx}} \sim \mathrm{A}_{\mathrm{yy}} \sim 6.0 \pm 0.2 \mathrm{mT}$ and $\mathrm{A}_{\mathrm{zz}}$ $\sim 7.2 \pm 0.2 \mathrm{mT}$. The spectra in between $40^{\circ}$ and $45^{\circ}$ are 
TABLE I. Principal values (in $\mathrm{mT}$ ) of the hf tensors for two pairs of ${ }^{27} \mathrm{Al}$ neighbors $\left(\mathrm{Al}_{1}, \mathrm{Al}_{4}\right.$ and $\left.\mathrm{Al}_{2}, \mathrm{Al}_{3}\right)$ obtained from calculations for $\mathrm{V}_{\mathrm{N}}^{0}$ and estimated from EPR for the EI- 1 defect. $\theta$ and $\varphi$ are the polar and azimuthal angles measured in degree, respectively, of the principal axes of the A tensors. The difference values for atoms in the same pair are due to error in the calculation $(\sim 0.3 \mathrm{mT})$. The error of the A-values estimated from EPR is in the similar range.

\begin{tabular}{lcccccc}
\hline \hline Center & Atom & $\mathrm{A}_{\mathrm{xx}}$ & $\mathrm{A}_{\mathrm{yy}}$ & $\mathrm{A}_{\mathrm{zz}}$ & $\theta$ & $\varphi$ \\
\hline $\mathrm{V}_{\mathrm{N}}^{0}$ & $\mathrm{Al}_{1}$ & 6.7 & 6.7 & 8.2 & 44.0 & 90.0 \\
& $\mathrm{Al}_{2}$ & 5.9 & 6.0 & 7.5 & 89.3 & -177.0 \\
& $\mathrm{Al}_{3}$ & 5.9 & 6.0 & 7.6 & 90.9 & 177.2 \\
& $\mathrm{Al}_{4}$ & 6.4 & 6.5 & 8.0 & 20.6 & 90.1 \\
\multirow{2}{*}{ EI-1 } & $\mathrm{Al}_{1-4}$ & $\sim 6.0$ & $\sim 6.0$ & $\sim 7.2$ & $\sim 40-45$ & \\
\hline \hline
\end{tabular}

rather similar and can be well fitted with the same hf parameters. The direction of the principal axes of hf tensors of the two Al pairs should be close to $\sim 40^{\circ}-45^{\circ}$ off the c-axis. The estimated hf parameters are given in Table I. The hf structures at intermediate angles can also be fitted assuming $C_{3 v}$ symmetry for the center with the hf splitting varying in the same range $(6.0-7.0 \mathrm{mT})$. However, the hf interaction with four nearly equivalent $\mathrm{Al}$ neighbors is typical for a defect with $C_{1 h}$ and not $C_{3 v}$ symmetry.

Since the EI-1 spectrum was only observed after electron irradiation, the associated defect is likely to be intrinsic. The hf interaction with four $\mathrm{Al}$ neighbors indicates that the defect is at $\mathrm{N}$ site and can be related to the $\mathrm{N}$ vacancy. With the effective electron spin $S=1 / 2$, the charge state of the $\mathrm{N}$ vacancy is expected to be neutral. However, $V_{N}^{0}$ has already been suggested as a possible model for another EPR center with $C_{3 v}$ symmetry by Evans et al. ${ }^{8}$

We modeled $V_{N}$ in a 432-atom hexagonal AlN supercell using a $\Gamma$-point sampling of the Brillouin zone. This model worked very well for other defects in AlN. ${ }^{9}$ We applied density functional theory calculations by choosing the PerdewBurke-Ernzerhof (PBE) functional. ${ }^{10}$ Plane waves with a cut-off of $420 \mathrm{eV}$ were utilized for the valence electrons together with PAW potentials. ${ }^{11}$ The geometry optimization was carried out by VASP $\operatorname{code}^{12}$ while the hf tensors were determined by the CPPAW code. ${ }^{13}$ The $\mathrm{V}_{\mathrm{N}}$ defect introduces levels in the fundamental band gap. The corresponding states show $a_{1}, a_{1}$, and $e$ characters in the unrelaxed case under $C_{3 v}$ point group where the first $a_{1}$ level is an $s$-like whereas the second $a_{1}$ and $e$ levels are $p$-like contribution of the Al dangling bonds split in the hexagonal crystal field. In the neutral charge state the first $a_{1}$ level is fully occupied and it is very close to the valence band edge. Because the calculated PBE band gap is too small $(4.0 \mathrm{eV})$, therefore the second $a_{1}$ and the $e$ levels are relatively close to the conduction band (CB) edge. However, these states are very localized and distinct from the delocalized $\mathrm{CB}$ states, so they presumably represent deep levels in the fundamental band gap. The second $a_{1}$ level is singly occupied in the neutral charge state, so $\mathrm{V}_{\mathrm{N}}^{0}$ is paramagnetic. If $C_{3 v}$ point group is preserved during geometry optimization then the neighbor $\mathrm{Al}$ atom of the vacancy along the c-axis moves farther from the vacant site. As a consequence, the spin density will be primarily localized on the dangling bond of this single Al-atom while the rest is mostly distributed among the other three neighbor Al-atoms in the basal plane. The singly occupied second $a_{1}$ level and the empty $e$ levels are separated by about $0.5 \mathrm{eV}$, so this $a_{1}$ level will be clearly separated from the $\mathrm{CB}$ edge by about $0.7 \mathrm{eV}$ even in PBE calculation. This configuration is metastable. We found that if two Al-atoms in the basal plane move closer to each other by distorting the symmetry of the defect to $C_{1 h}$ then the spin density distribution among the four Al-atoms near the vacant site will be almost equal. During this pairwise reconstruction the distances between the closest second neighbor $\mathrm{Al}$ atoms are around $2.94 \AA$. In this case the singly occupied $a^{\prime}$ level shifts down in the gap by about $0.23 \mathrm{eV}$ compared to the counterpart $a_{1}$ level in $C_{3 v}$ configuration which stabilizes the $C_{1 h}$ configuration over the $C_{3 v}$ configuration by about $0.14 \mathrm{eV}$. This is a significant energy difference, thus we provide the hf tensors of neighbor ${ }^{27} \mathrm{Al}$ isotopes only for the most stable $C_{1 h}$ configuration.

As can be seen in Table I, the calculated principal ${ }^{27} \mathrm{Al}$ hf values are in good agreement with the experimental values estimated from EPR for the EI-1 defect. These hf parameters are completely different from that obtained for the $C_{3 v}$ center by Evans and co-workers ${ }^{8}\left(\mathrm{~A}_{\|}=111.3 \mathrm{MHz} \sim 3.97 \mathrm{mT}\right.$ and $\mathrm{A}_{\perp}=54.19 \mathrm{MHz} \sim 1.93 \mathrm{mT}$ for the $\mathrm{Al}$ atom long the c-axis and much smaller for other $\mathrm{Al}$ atoms in the basal plane). We therefore suggest the EI-1 defect to be the better candidate for the neutral $\mathrm{N}$ vacancy in AlN.

Following the one-electron linear-combination of atomic-orbital approximation, we estimate the isotropic part $a$ and the anisotropic part $b$ of the hf $\mathrm{A}$ tensor to be: $a$ $\sim 179.3 \mathrm{MHz}$ and $b \sim 11.2 \mathrm{MHz}$. These correspond to the spin density on the $s$ and $p$ orbitals of four Al neighbors of $\sim 18 \%$ and $\sim 54 \%$, respectively, or $\sim 72 \%$ in total.

In summary, we have observed the EI-1 EPR spectrum in electron-irradiated AlN. The spectrum shows a clear hf structure due to the interaction with four nearest Al neighbors. Based on the good agreement in the hf parameters estimated from EPR and obtained from $a b$ initio supercell calculations for $\mathrm{V}_{\mathrm{N}}^{0}$, we suggest the EI-1 defect to be the best candidate for the neutral $\mathrm{N}$ vacancy in AlN. The high spin localization suggests that the defect is a deep center.

Support from the Swedish Energy Agency, the Swedish Foundation for Strategic Research, the Swedish Research Council, the Knut and Alice Wallenberg Foundation, Hungarian OTKA Grant No. K-67886, NHDP TÁMOP-4.2.1/B09/1/KMR-2010-0002 program and the Swedish National Infrastructure for Computing is acknowledged.

\footnotetext{
${ }^{1}$ Y. Taniyasu, M. Kasu, and T. Makimoto, Nature (London) 441, 325 (2006).

${ }^{2}$ I. Gorczyca, A. Svane, and N. E. Christensen, Phys. Rev. B 60, 8147 (1999).

${ }^{3}$ A. Fara, F. Bernardini, and V. Fiorentini, J. Appl. Phys. 85, 2001 (1999).

${ }^{4}$ C. Stampfl and C. G. Van de Walle, Phys. Rev. B 65, 155212 (2002).

${ }^{5}$ K. Atobe et al., Jpn. J. Appl. Phys., Part 1 29, 150 (1990).

${ }^{6}$ M. Honda et al., Jpn. J. Appl. Phys., Part 2 29, L652 (1990).

${ }^{7}$ P. M. Mason et al., Phys. Rev. B 59, 1937 (1999).

${ }^{8}$ S. M. Evans et al., Appl. Phys. Lett. 88, 062112 (2006).

${ }^{9}$ Á. Szabó et al., Appl. Phys. Lett. 96, 192110 (2010).

${ }^{10}$ J. P. Perdew et al., Phys. Rev. Lett. 77, 3865 (1996).

${ }^{11}$ P. E. Blöchl, Phys. Rev. B 50, 17953 (1994).

${ }^{12}$ G. Kresse and J. Hafner, Phys. Rev. B 49, 14251 (1994).

${ }^{13}$ P. E. Blöchl, C. J. Först, and J. Schimpl, Bull. Mater. Sci. 26, 33 (2003)
} 